\title{
CodY-affected transcriptional gene expression of Streptococcus pyogenes during growth in human blood
}

\author{
Correspondence \\ Horst Malke \\ horst-malke@ouhsc.edu
}

Received 4 October 2006

Accepted 27 January 2007

\author{
Horst Malke and Joseph J. Ferretti
}

Oklahoma University Health Sciences Center, Department of Microbiology and Immunology, Oklahoma City, OK 73190, USA

\begin{abstract}
In an attempt to expand the available knowledge of pathogen-host interactions during ex vivo growth of Streptococcus pyogenes (GAS) in nonimmune whole human blood, the extents to which the expression of 51 genes including regulators with known targets, established virulence factors, physiologically important transporters and metabolic enzyme genes was differentially affected in the presence or absence of a functional $\operatorname{cod} Y$ gene were determined. The results obtained by quantitative real-time PCR using the M49 strain NZ131 showed that CodY influenced GAS gene activity in a dynamic fashion, with differential responses detected for 26 genes and occasionally characterized by discordance in the blood environment compared to laboratory medium. Degenerate derivatives of the recently discovered CodY box potentially serving as a cis-regulatory element for CodY action were identified in the upstream regions of 15 genes of the NZ131 genome, and these genes featured sequence motifs identical to the NZ131 CodY box in all completely sequenced $S$. pyogenes genomes. As none of these genes represented a genuine virulence factor, it seems likely, therefore, that the observed differential transcription of the majority of virulence genes was caused by indirect actions of CodY as part of a regulatory network.
\end{abstract}

\section{INTRODUCTION}

Streptococcus pyogenes (group A streptococcus, GAS) is a human-specific pathogen that causes high morbidity characterized by many types of skin and upper respiratory tract infections. The organism has also attained notoriety by causing occasionally life-threatening invasive disease characterized by deep tissue or bloodstream infections. Although GAS has long been known to be capable of multiplying in non-opsonizing blood and several virulence factors contributing to immune evasion have been identified (Cunningham, 2000), the full spectrum of bacterially encoded regulatory proteins mediating pathogen-host interactions remains elusive. Recently, has $A B C$-encoded hyaluronic acid capsule synthesis has been shown to be rapidly induced in the bloodstream of GAS-infected mice but the nature of the stimulus remained unknown (Gryllos et al., 2001). During the initial period of growth in human blood, transcription of the two-component regulatory system CovRS, which negatively affects has expression

\section{Abbreviation: GAS, group A streptococcus.}

Tables showing the targets and primers used for real-time reverse transcription PCR and the mean transcript values of the genes and strains are available as supplementary material with the online version of this paper.
(Federle et al., 1999), increases but transcript levels return to normal $30 \mathrm{~min}$ after adaptation to blood (Graham et al., 2005). Moreover, the latter comprehensive study of GAS transcriptome dynamics during growth in human blood provided ample evidence for an important role of the CovRS system in the remodelling of GAS metabolism and virulence-associated functions in this environment (Graham et al., 2005).

In previous studies, the CodY protein, highly conserved in the low-G $+C$ Gram-positive bacteria, has been identified as a pleiotropic transcriptional regulator that is activated by branched-chain amino acids and controls many genes which become operative when the cells face nutrient limitation (den Hengst et al., 2005; Guédon et al., 2005; Levdikov et al., 2006; Malke et al., 2006; Sonenshein, 2005). In S. pyogenes grown in Todd-Hewitt broth or chemically defined medium, CodY affects, directly or indirectly, the expression of certain regulatory genes, virulence factors, transporters and metabolic enzyme genes, often in a medium- and nutritional status-dependent fashion (Malke et al., 2006). To expand these observations to include an environment with clinical relevance, we examined the same functional categories of genes during cultivation of $S$. pyogenes in whole human blood and show here that CodY influences not only covRS expression in a dynamic 
fashion but similar to CovR has a much broader role in structuring GAS gene activity in an environment mimicking streptococcal septicaemia.

\section{METHODS}

Bacterial strains and culture conditions. S. pyogenes strain NZ131 (M type 49) and its codY1 mutant were used throughout this study. The codY1 mutation, constructed by nonpolar insertion mutagenesis and confirmed as described previously (Malke et al., 2006), disrupted $\operatorname{cod} Y$ at codon 197, implying that the sequence segment encoding the helix-turn-helix DNA-binding motif (Levdikov et al., 2006) was removed from the $3^{\prime}$-portion of the truncated $\operatorname{cod} Y$ gene. Cells were grown statically at $37{ }^{\circ} \mathrm{C}$ in Todd-Hewitt broth containing $0.2 \%$ yeast extract (THY broth) or fresh heparinized nonimmune whole human blood drawn on different occasions from a healthy 68-yearold male individual according to a protocol approved by the Institutional Review Board for Human Subjects. The blood was used without previous heat treatment to preserve complement components. Seventeen millilitres of mid-exponential phase THY cultures grown to $\mathrm{OD}_{600} 0.6$ were centrifuged and cells were resuspended at zero time in the same volume of blood at a multiplicity of $\sim 10$ c.f.u. per leukocyte. At $0,1,2$ and $3 \mathrm{~h}$ of incubation, samples were taken for colony counts on blood agar plates, and $5 \mathrm{ml}$ aliquots were withdrawn and added to $13 \mathrm{ml}$ RNAlater (Ambion) for RNA stabilization.

RNA isolation, cDNA synthesis and quantitative real-time PCR. To lyse erythrocytes, $25 \mathrm{ml}$ EL buffer (Qiagen) was added and the mixtures were incubated on ice for $20 \mathrm{~min}$. After centrifugation at $6000 \mathrm{~g}$ and washing with EL buffer, bacterial cells were subjected to total RNA isolation with subsequent $\mathrm{rDNase}$ I treatment by the RiboPure-Bacteria Protocol (Ambion) as previously described in detail (Malke et al., 2006). RNA integrity was assessed electrophoretically (Malke et al., 2006). cDNA synthesis from total RNA with random primers, dNTPs and Superscript II reverse transcriptase (Invitrogen) followed by RNase A and RNase $\mathrm{H}$ treatment has also been detailed previously (Malke et al., 2006). The amounts of genespecific cDNAs were quantified by the comparative $C_{T}\left(\Delta \Delta C_{T}\right)$ method using the Bio-Rad MyiQ Real-Time PCR Detection system with fluorescein-spiked SYBR Green as fluorophore (Malke et al., 2006). PCR primers specified in Supplementary Table S1 in the online version of this paper were designed as described before (Malke et al., 2006). Primer specificity was verified by running amplification plus melt-curve protocols for every target gene and experimental condition. Expression fold changes of the genes of interest were normalized for both strains to the constitutively expressed gyrA gene, which served as endogenous reference (see Supplementary Table S2 in the online version of this paper). Quantitative real-time PCR was performed in duplicate on RNA purified from two to four independently grown cultures. The significance of fold changes of gene expression in the mutant relative to the wild-type strain was assessed by determining statistical probabilities based on Student's two-tailed $t$-test, with $P \leqslant 0.0500$ being considered significant.

\section{RESULTS AND DISCUSSION}

\section{Gene expression analysis}

c.f.u. counts show that both wild-type and the codY1 mutant strains were capable of multiplication during exposure to blood, with any differences between them not attaining significance. This observation is relevant because it rules out the possibility that differential gene expression was attributable to differences in growth rates between the two strains. Growth rates were highest during the first hour of growth and then declined such that c.f.u. numbers increased by a factor of $\sim 3.2$ within the $3 \mathrm{~h}$ incubation period (Fig. 1).

A total of 51 genes were compared between the wild-type and the $\operatorname{cod} Y$ mutant for ex vivo expression in blood using quantitative real-time PCR (Table 1). These genes included transcription factors with known targets, prominent virulence-associated genes and transporters as well as metabolic enzyme genes physiologically important to this polyauxotrophic pathogen. Compared to the trendier but inherently more variable microarray technique, this approach limited the number of genes assayed but was considered more efficient and reliable in detecting lowabundance transcripts (Graham et al., 2002), and in yielding significant transcription differences with expression fold changes smaller than two (Table 1). Of the 51 genes examined, the transcription of 26 genes representing 23 transcription units was differentially affected in the two strains. Among the transcription factors, $\operatorname{cod} Y$ transcript levels, measured by PCR primers targeting the expressed 5' segment of the truncated codY1 allele (Malke et al., 2006), were most strongly affected, with derepression in the mutant being highest during the first hour of blood culturing. Derepression of codY activity prevailed at a lower level during the next $2 \mathrm{~h}$ of incubation, an observation accountable for by the exhaustion of free branched-chain amino acids required for full CodY activity (Guédon et al., 2001; Shivers \& Sonenshein, 2004). Similar to codY mutant cells grown in THY broth, those cultured in blood expressed the two-component regulatory systems CovRS and SptRS at higher levels than the wild-type. In the case of $\operatorname{cov} R S$, upregulation decreased with increasing time of incubation in blood, again suggestive of decreasing CodY

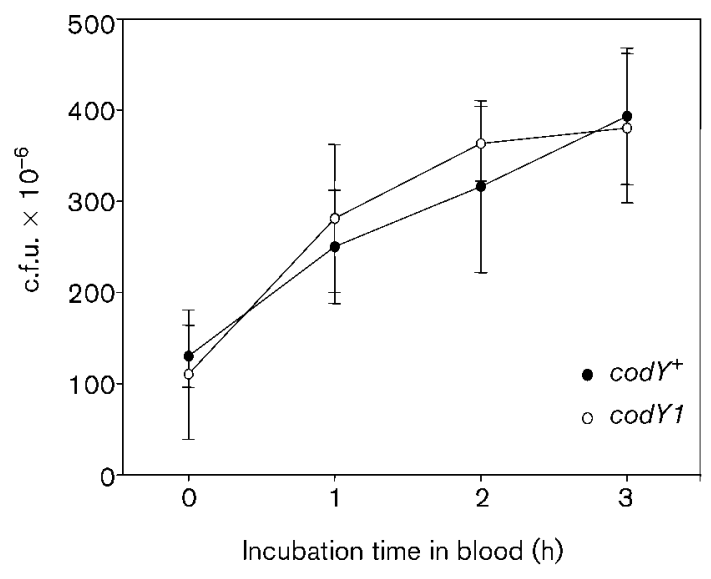

Fig. 1. Growth of NZ131 wild-type and NZ131 cod $Y 1$ in human blood. Error bars indicate standard deviations calculated from four independent experiments using blood drawn on four different occasions. 
Table 1. CodY-dependent gene expression in S. pyogenes strain NZ131 grown in human blood

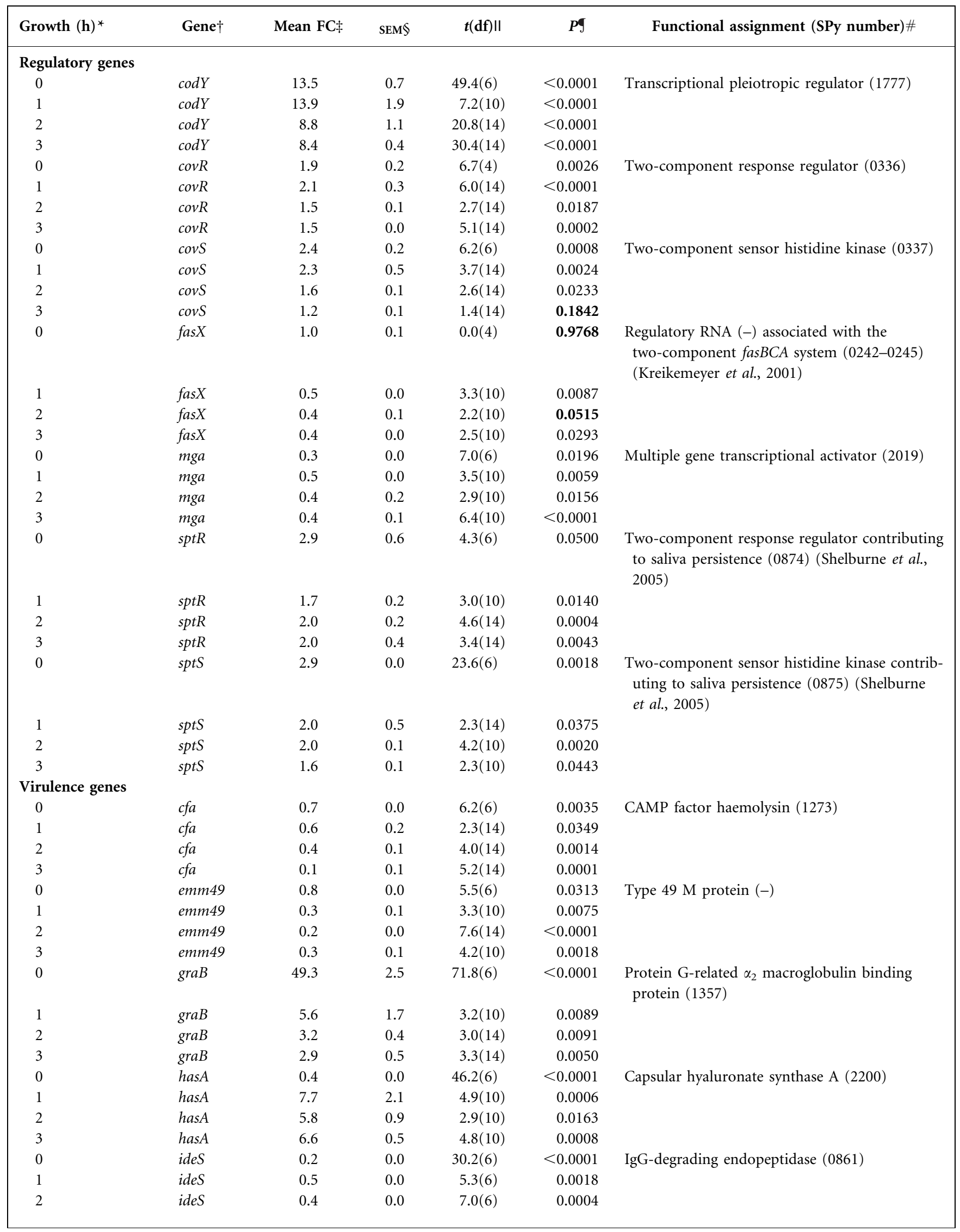


Table 1. cont.

\begin{tabular}{|c|c|c|c|c|c|c|}
\hline Growth $(h)^{*}$ & Gene $\dagger$ & Mean FC $\ddagger$ & SEM\$ & $t(\mathrm{df}) \|$ & $P \mathbb{G}$ & Functional assignment (SPy number) \# \\
\hline 3 & ides & 0.7 & 0.0 & $6.7(6)$ & 0.0006 & \\
\hline 0 & $n g a$ & 0.5 & 0.1 & $6.3(6)$ & 0.0008 & NAD glycohydrolase $(0165)$ \\
\hline 1 & nga & 0.5 & 0.1 & $4.6(14)$ & 0.0004 & \\
\hline 2 & $n g a$ & 0.4 & 0.1 & $7.5(14)$ & $<0.0001$ & \\
\hline 3 & $n g a$ & 0.3 & 0.1 & $5.0(10)$ & 0.0005 & \\
\hline 0 & prtS & 0.04 & 0.0 & $23.3(6)$ & $<0.0001$ & Cell surface proteinase $(0416)$ \\
\hline 1 & prtS & 0.5 & 0.1 & $5.1(10)$ & 0.0004 & \\
\hline 2 & prtS & 0.5 & 0.1 & $9.4(10)$ & $<0.0001$ & \\
\hline 3 & prtS & 1.2 & 0.2 & $0.4(10)$ & 0.6978 & \\
\hline 0 & $s c l$ & 0.03 & 0.0 & $16.5(6)$ & $<0.0001$ & Collagen-like surface protein (1054) \\
\hline 1 & scl & 0.1 & 0.0 & $4.3(10)$ & 0.0016 & \\
\hline 2 & $s c l$ & 0.2 & 0.0 & $12.9(10)$ & $<0.0001$ & \\
\hline 3 & $s c l$ & 0.3 & 0.1 & $2.4(10)$ & 0.0404 & \\
\hline 0 & $\operatorname{scp} A$ & 0.1 & 0.0 & $28.5(6)$ & $<0.0001$ & Complement C5a peptidase (2010) \\
\hline 1 & $\operatorname{scp} A$ & 0.2 & 0.0 & $11.7(10)$ & $<0.0001$ & \\
\hline 2 & $\operatorname{scp} A$ & 0.2 & 0.0 & $10.7(10)$ & $<0.0001$ & \\
\hline 3 & $\operatorname{scp} A$ & 0.7 & 0.0 & $7.8(6)$ & 0.0002 & \\
\hline 0 & $s k a$ & 3.9 & 0.6 & $6.3(6)$ & 0.0007 & Streptokinase (1979) \\
\hline 1 & $s k a$ & 0.4 & 0.1 & $3.1(10)$ & 0.0120 & \\
\hline 2 & $s k a$ & 0.8 & 0.1 & $2.3(10)$ & 0.0436 & \\
\hline 3 & $s k a$ & 1.5 & 0.2 & $1.3(10)$ & 0.2401 & \\
\hline 0 & slo & 0.6 & 0.1 & $3.7(6)$ & 0.0104 & Streptolysin O (0167) \\
\hline 1 & slo & 0.3 & 0.0 & $3.5(14)$ & 0.0034 & \\
\hline 2 & slo & 0.3 & 0.1 & $7.8(14)$ & $<0.0001$ & \\
\hline 3 & slo & 0.4 & 0.1 & $3.7(10)$ & 0.0039 & \\
\hline 0 & sof & 0.6 & 0.0 & $23.4(6)$ & $<0.0001$ & Serum opacity factor $(-)$ \\
\hline 1 & sof & 0.3 & 0.1 & $5.9(10)$ & 0.0002 & \\
\hline 2 & sof & 0.3 & 0.0 & $2.6(10)$ & 0.0264 & \\
\hline 3 & sof & 1.0 & 0.0 & $0.0(6)$ & 0.9673 & \\
\hline 0 & speH & 0.2 & 0.0 & $8.9(6)$ & 0.0009 & Pyrogenic exotoxin H (1008) \\
\hline 1 & speH & 0.3 & 0.0 & $11.7(6)$ & $<0.0001$ & \\
\hline 2 & speH & 0.3 & 0.0 & $69.2(6)$ & $<0.0001$ & \\
\hline 3 & speH & 0.6 & 0.0 & $6.2(6)$ & 0.0008 & \\
\hline \multicolumn{7}{|l|}{ Transporters } \\
\hline 0 & braB & 40.6 & 1.2 & $63.2(6)$ & $<0.0001$ & $\begin{array}{l}\text { Branched-chain amino acid transport protein } \\
(0323)\end{array}$ \\
\hline 1 & braB & 5.1 & 1.5 & $3.9(10)$ & 0.0031 & \\
\hline 2 & braB & 4.6 & 0.7 & $3.8(10)$ & 0.0038 & \\
\hline 3 & $b r a B$ & 4.9 & 1.3 & $3.9(10)$ & 0.0028 & \\
\hline 0 & $d p p A$ & 0.3 & 0.0 & $12.2(6)$ & 0.0003 & $\begin{array}{l}\text { Dipeptide } A B C \text { transporter substrate binding } \\
\text { protein }(2000)\end{array}$ \\
\hline 1 & $d p p A$ & 0.7 & 0.1 & $1.3(14)$ & 0.2029 & \\
\hline 2 & $d p p A$ & 0.7 & 0.0 & $2.5(14)$ & 0.0250 & \\
\hline 3 & $d p p A$ & 0.9 & 0.0 & $2.1(14)$ & 0.0509 & \\
\hline 0 & $p t s G$ & 0.6 & 0.2 & $1.8(6)$ & 0.1452 & $\begin{array}{l}\text { Enzyme II of glucose-specific phosphotransferase } \\
\text { system (1986) }\end{array}$ \\
\hline 1 & $p t s G$ & 3.1 & 0.6 & $2.8(14)$ & 0.0147 & \\
\hline 2 & $p t s G$ & 3.4 & 0.5 & $4.1(14)$ & 0.0011 & \\
\hline 3 & $p t s G$ & 2.9 & 0.6 & $3.5(14)$ & 0.0038 & \\
\hline 0 & pyrP & 0.8 & 0.1 & $2.9(6)$ & 0.0463 & Uracil permease (0831) \\
\hline 1 & pyrP & 0.4 & 0.1 & $6.0(10)$ & 0.0001 & \\
\hline 2 & pyrP & 1.3 & 0.2 & $0.6(14)$ & 0.5642 & \\
\hline 3 & pyrP & 1.1 & 0.1 & $0.4(14)$ & 0.7215 & \\
\hline \multicolumn{7}{|c|}{ Metabolic enzymes } \\
\hline 0 & $\operatorname{arc} A$ & 0.6 & 0.1 & $4.6(6)$ & 0.0101 & Arginine deiminase (1547) \\
\hline 1 & $\operatorname{arc} A$ & 6.5 & 1.8 & $6.8(6)$ & 0.0005 & \\
\hline
\end{tabular}


Table 1. cont.

\begin{tabular}{|c|c|c|c|c|c|c|}
\hline Growth $(h)^{*}$ & Gene $\dagger$ & Mean FC $\ddagger$ & SEM $\$$ & $t(\mathrm{df}) \|$ & $P \mathbf{g}$ & Functional assignment (SPy number) \# \\
\hline 2 & $\operatorname{arc} A$ & 7.0 & 0.8 & $17.4(6)$ & $<0.0001$ & \\
\hline 3 & $\operatorname{arc} A$ & 1.6 & 0.3 & $2.9(6)$ & 0.0281 & \\
\hline 1 & $\operatorname{asn} A$ & 1.7 & 0.1 & $5.1(6)$ & 0.0022 & \\
\hline 2 & $\operatorname{asn} A$ & 1.8 & 0.1 & $25.2(6)$ & $<0.0001$ & \\
\hline 3 & $\operatorname{asn} A$ & 1.2 & 0.1 & $1.6(6)$ & 0.1690 & \\
\hline
\end{tabular}

*Zero hour growth point corresponding to mid-exponential phase cells $\left(\mathrm{OD}_{600} 0.6\right)$ precultivated in THY broth.

$\dagger$ The following genes (SPy numbers) not included in Table 1 showed insignificant differential expression at any time point during blood culture of the two strains. Regulatory genes: $c c p A$ (0514), fasA (0245), msmR (-), phoU (1240), pyrR (0830), relA (1981), ropB (2042); virulence genes: sagA (0738), sagB (0739), sda (2043), speB (2039); transporters: malX (1294), oppA (0293), oppB (0294), pbuX (1137), potA (1102), pstB (1241), scrA (1815); metabolic enzyme genes: $d t d$ (1980), $g \ln A$ (1877), nadE (1652), pepB (1393), pepF (0606), pncA (1776) and xpt (1136).

$\$$ FC, Expression fold change in NZ131 codY1 relative to wild-type NZ131.

\$SEM, Standard error of the mean expression fold change.

IIt(df), Student's $t$ value (including the degree of freedom).

I $P, P$ value based on Student's $t$-test, with figures in bold indicating insignificant fold changes.

\#SPy numbers based on annotation of the SF370 (M type 1) genome (Ferretti et al., 2001). A dash indicates that no SPy number has been assigned because the gene is absent from SF370 or assignment is pendent.

activity during the course of incubation. Contrary to the aforementioned regulatory genes, the multigene activators $m g a$ and fasX were downregulated in NZ131 codY1, an effect not observed for fasX in cells grown in THY broth. The failure to find fasA to be downregulated as well may be explained by the observation that fas $X$ shows strong independent transcription from the fas $B C A$ members of the fas operon (Kreikemeyer et al., 2001; Steiner \& Malke, 2001, 2002). Thus CodY-mediated positive control of fasX transcription appears to depend on the blood environment and to be directed toward the independent mode of transcription of the terminal member of the fas operon.
This finding is important in view of the fact that FasX was found to stimulate streptococcal aggressiveness towards host cells by promoting cell adherence and internalization, cytokine expression and release, as well as host cell apoptosis (Klenk et al., 2005).

Consistent with underexpression of mga in the codY1 mutant strain, virulence genes known to be activated by Mga (emm49, scl, scpA, sof) (Hynes, 2004) were strongly downregulated in blood as well, with underexpression tending to become less severe, or even absent in the case of sof, towards the end of the exposure time. Additional

Table 2. NZ131 genes displaying a CodY motif in their upstream region

\begin{tabular}{|lllcl|}
\hline SPy number & Gene & \multicolumn{1}{c|}{ Motif $\dagger$} & Position/ATG $\ddagger$ & \multicolumn{1}{c|}{ (Putative) Functional assignment } \\
\hline 0293 & $o p p A$ & AATaTTCAGAtAATT & -110 & Oligopeptide periplasmic binding protein \\
0323 & $b r a B$ & AATTTTCAGtcAATT & -151 & Branched-chain amino acid transport protein \\
0647 & $b u d C$ & tAgTTTCAGAAAATa & -152 & Acetoin reductase \\
0870 & $f m s$ & AATaTTCAGAAAATT & -385 & Formylmethionine deformylase \\
1414 & - & AATTTTCTGAAAAag & -78 & Potassium uptake protein \\
1654 & $a a p A$ & AATTTTCTGAAAAaa & -38 & Amino acid permease \\
1676 & $t k t$ & gATTTTCTGAAAAag & -73 & Transketolase \\
1738 & $m a n L$ & AtTTTTCAGAAAATg & -320 & Mannose-specific phosphotransferase component IIAB \\
1758 & $p h a B$ & AATTaTCAGAAAAga & -8 & Enoyl-CoA hydratase \\
1777 & $\operatorname{codY}$ & AATTTTCTGAtAATT & -66 & Transcriptional pleiotropic regulator \\
1825 & $u v r A$ & gATTTTCTGAAAAaT & -85 & UvrABC system protein A \\
1827 & - & AtTTTTCAGAAAATc & -71 & Divalent cation (Mg ++ \\
1881 & $p g k$ & tATTTTCTGAAAATa & -117 & Phosphoglycerate kinase \\
2150 & argR & AtTTTTCTGAAAATg & -259 & Arginine repressor \\
2151 & argS & cATTTTCAGAAAAaT & -118 & Arginyl-tRNA synthetase \\
& & & & \\
\hline
\end{tabular}

${ }^{\star}$ Based on annotation of the SF370 (M type 1) genome (Ferretti et al., 2001).

$\nmid$ Lower-case letters indicate mismatches from the consensus sequence AATTTTCWGAAAATT (den Hengst et al., 2005; Guédon et al., 2005). ‡Distance (number of bases) of the CodY motif from the translational start codon. 
prominent virulence factors showing decreased transcript levels in the mutant included $c f a$, ideS, nga, slo, ska and speH, genes often influenced in an incubation time-dependent fashion. Of the latter, ska and ideS are under CovR control (Federle et al., 1999; Hynes 2004) and apparently were negatively affected by the increased CovR levels in the mutant. Additionally, decreased levels of FasX in the codY1 mutant may contribute to the downregulation of $s k a$, which is under positive FasX control (Kreikemeyer et al., 2001). Of relevance, CodY-mediated stimulation of $s k a$, slo and ideS expression implicates this regulator in the positive control of genes encoding nutrient-releasing proteins, thus tying in with a role in not only sensing nutrient availability but also contributing to nutrient release. In contrast to the majority of the virulence genes with lower activity, two important virulence factors, graB and hasA, showed highly increased transcript levels in the $\operatorname{cod} Y 1$ mutant. Interestingly, for has $A$, this behaviour was discordant with transcription in THY broth but similar derepression of the has operon has been observed in a $\operatorname{cov} R$ mutant during growth in blood (Graham et al., 2005), consistent with negative transcriptional control of the has genes by CovR.

Transporters strongly upregulated during codY1 mutant growth in blood included $b r a B$ and ptsG. Of special relevance is $b r a B$, which is also under strong negative stringent control during isoleucine plus valine starvation (Malke et al., 2006). This shows that the wild-type, in repressing braB during growth in complex media, exploits a nutritional status-specific, cost-effective means for the abrogation of futile transport processes. Repression in the wild-type of glucose transport ( $p t s G$ ) as well as temporal repression of amino acid synthesis or amino acid catabolic enzymes (asnA and $\operatorname{arc} A$, respectively) may be regarded as examples of the involvement of CodY in the control of basic metabolic processes during growth in blood. Concerning arginine deiminase encoded by $\operatorname{arcA}$, this enzyme, by releasing ammonia from arginine, might also support the survival of $S$. pyogenes under acidic conditions such as those present in phagolysosomes (Degnan et al., 2000).

\section{Identification of the CodY box in NZ131}

A 15-nucleotide palindromic sequence (AATTTTCWGAAAATT) serving as a high-affinity binding site for CodY has recently been identified in Lactococcus lactis (den Hengst et al., 2005; Guédon et al., 2005). Derivatives of the CodY box with high similarity scores were also detected by in silico analysis in the upstream regions of genes from other Grampositive bacteria (den Hengst et al., 2005; Guédon et al., 2005), including five genes from S. pyogenes (Guédon et al., 2005). Using BLAST searches (www.ncbi.nlm.nih.gov) against the genomic sequence of NZ131 and a threshold value of $80 \%$ sequence identity (three mismatches accepted), we detected the CodY box in the upstream regions of 15 NZ131 genes. These included $\operatorname{cod} Y, b r a B$ and $o p p A$ (Table 2), genes also found by Guédon et al. (2005) in strains of M types other than M49. While codY and braB were strongly responsive to CodY repression in the present experiments (Table 1), $o p p A B$ repression was evident only in laboratory media (Table 1, footnote†; Malke et al., 2006). This provides another example of environmentally dependent gene responses to the action of CodY. As codY is transcribed both monocistronically and dicistronically from the cooriented upstream aat gene encoding aspartate aminotransferase (Malke et al., 2006), it is interesting to point out that the CodY box precedes only the coding region of $\operatorname{cod} Y$ but not aat. This suggests that negative transcriptional autoregulation of $\operatorname{cod} Y$ applies only to monocistronic transcription of this gene. The dicistronic mode adds a strong autonomous component to $\operatorname{cod} Y$ expression, a phenomenon not observed in the nonpathogenic Bacillus subtilis and $L$. lactis species (den Hengst et al., 2005; Guédon et al., 2005; Sonenshein, 2005). Confirming this notion, the results of previous experiments indicated that aat is not under the control of CodY (Malke et al., 2006), and the same situation applies to the organization and expression pattern of the aat and $\operatorname{cod} Y$ genes from the M1 strain SF370 (Ferretti et al., 2001; Malke et al., 2006). This situation may have developed to facilitate growth of this fastidious organism under various environmental conditions.

Table 2 shows that the majority of genes carrying the CodY box encoded regulators, transporters or proteins involved in nitrogen and carbon metabolism. The genes with CodY motifs detected in NZ131 were also present in all GAS genomes accessible at www.ncbi.nlm.nih.gov. As an interesting detail, the CodY box precedes the coding sequence of $\mathrm{a} \mathrm{Mg}^{++}$transport protein (Spy1827). Since the CovS sensor histidine kinase is specifically activated by environmental magnesium (Gryllos et al., 2003), these observations may provide a clue as to how the CodY and CovRS systems interact during GAS growth in human body fluids, which contain low magnesium concentrations (Gryllos et al., 2003): CodY-mediated repression of magnesium uptake would place the CovRS system in an inactive state, resulting in the derepression of virulence factors controlled by CovR. There were also two instances where the CodY box was located in the intergenic region between adjacent genes with opposite orientations. Most interestingly, these included $\operatorname{argR}$ and $\operatorname{argS}$, strongly suggesting that the arginine repressor and arginyl-tRNA synthetase are coregulated by CodY. Notably, all virulence factors responsive to CodY action in this and a previous study (Table 1; Malke et al., 2006) lacked the CodY box. In line with this observation, a substantial proportion of the known CodY target genes of $B$. subtilis and $L$. lactis do not display the CodY box (den Hengst et al., 2005; Guédon et al., 2005). In the same vein, the most differentially expressed $S$. pyogenes genes responsive to CovR action do not reveal a conserved sequence motif in their regulatory region (Federle \& Scott, 2002; Graham et al., 2002). Thus higher levels of degeneracy of the CodY box, higher order DNA structures enabling CodY binding, or, most likely, indirect effects caused by the amplification of CodY action through its interaction with other regulators may contribute to the broad action of this 
regulator (den Hengst et al., 2005; Guédon et al., 2005; Malke et al., 2006; Serror \& Sonenshein, 1996).

\section{Conclusions}

The present data provide strong evidence that the pleiotropic action of CodY observed in complex or defined laboratory media (Malke et al., 2006) extends to the human blood environment which, despite the experimental curtailment involved in ex vivo cultivation (Graham et al., 2005), is of greater clinical relevance than the artificial media. This is highlighted by our observation that transcriptional responses directed by CodY are not always concordant during growth in laboratory media versus growth in blood, suggesting the action of biological cues which are missing in THY broth or chemically defined medium. From the viewpoint of a more comprehensive understanding of virulence regulation, it is apparent that CodY contributes to the regulation of expression of key virulence genes. The observations that many virulence genes are upregulated in the wild-type following the initial phases of blood culture (Graham et al., 2005) but remain downregulated in the codY1 mutant (Table 1) strongly suggest that CodY contributes to the dynamics of gene expression during GAS growth in blood. As in the case of other pleiotropic regulators acting in GAS (Chaussee et al., 2004; Graham et al., 2002, 2005), more information is necessary to distinguish between direct and indirect CodY effects on gene expression. Genes showing the highest differential expression levels in the strain pair used here would appear to qualify as prime candidates for being direct $\operatorname{CodY}$ targets ( $\operatorname{cod} Y, \operatorname{braB}, \mathrm{graB}, s c l$ and $s c p A)$. However, this assumption seems to be coherent with the presence of the CodY box only for $\operatorname{cod} Y$ itself and $b r a B$. Concerning the majority of the virulence genes that show transcription differences between the wild-type and the $\operatorname{cod} Y$ mutant, it seems likely that indirect actions of $\operatorname{CodY}$ as component of a regulatory network are involved. In any case, any indirect CodY effects do not diminish the physiological role of CodY and its clinical relevance. A particularly interesting situation with the potential of shedding additional light on the interaction of CodY and CovR has recently been discovered by Gusa et al. (2007). They found that CovR stimulates $d p p A$ transcription in vivo but not in vitro using purified RNA polymerase and major sigma factor from GAS. As not only CovR but also CodY is required for activation of $d p p A$ transcription in vivo (Malke et al., 2006 and Table 1; Gusa et al., 2007), CodY might act as co-activator of CovR in promoting $d p p A$ expression (Gusa et al., 2007). Clearly, a $\operatorname{cod} Y-\operatorname{cov} R$ double mutant may help unravel the complex mechanism of $d p p A$ expression and possibly that of other multiply controlled GAS genes.

\section{ACKNOWLEDGEMENTS}

We thank C. Bennet for phlebotomy and M. McShan for providing the genomic sequence of strain NZ131 before publication. This work was supported by NIH/NIAID grant AI054473 to H. M.

\section{REFERENCES}

Chaussee, M. A., Callegari, E. A. \& Chaussee, M. S. (2004). Rgg regulates growth phase-dependent expression of proteins associated with secondary metabolism and stress in Streptococcus pyogenes. J Bacteriol 186, 7091-7099.

Cunningham, M. W. (2000). Pathogenesis of group A streptococcal infections. Clin Microbiol Rev 13, 470-511.

Degnan, B. A., Fontaine, M. C., Doebereiner, A. H., Lee, J. J., Mastroeni, P., Dougan, G., Goodacre, J. A. \& Kehoe, M. A. (2000). Characterization of an isogenic mutant of Streptococcus pyogenes Manfredo lacking the ability to make streptococcal acid glycoprotein. Infect Immun 68, 2441-2448.

den Hengst, C. D., van Hijum, S. A. F. T., Geurts, J. M. W., Nauta, A., Kok, J. \& Kuipers, O. P. (2005). The Lactococcus lactis CodY regulon: identification of a conserved cis-regulatory element. J Biol Chem $\mathbf{2 8 0}$, 34332-34342.

Federle, M. J. \& Scott, J. R. (2002). Identification of binding sites for the group A streptococcal global regulator CovR. Mol Microbiol 43, 1161-1172.

Federle, M. J., Mclver, K. S. \& Scott, J. R. (1999). A response regulator that represses transcription of several virulence operons in the group A streptococcus. J Bacteriol 181, 3649-3657.

Ferretti, J. J., McShan, W. M., Ajdic, D., Savic, D. J., Savic, G., Lyon, K., Primeaux, C., Sezate, S., Suvorov, A. N. \& other authors (2001). Complete genome sequence of an M1 strain of Streptococcus pyogenes. Proc Natl Acad Sci U S A 98, 4658-4663.

Graham, M. R., Smoot, L. M., Migliaccio, C. A., Virtaneva, K., Sturdevant, D. E., Porcella, S. F., Federle, M. J., Adams, G. J., Scott, J. R. \& Musser, J. M. (2002). Virulence control in group A streptococcus by a two-component gene regulatory system: global expression profiling and in vivo infection modeling. Proc Natl Acad Sci U S A 99, 13855-13860.

Graham, M. R., Virtaneva, K., Porcella, S. F., Barry, W. T., Gowen, B. B., Johnson, C. R., Wright, F. A. \& Musser, J. M. (2005). Group A Streptococcus transcriptome dynamics during growth in human blood reveals bacterial adaptive and survival strategies. Am J Pathol 166, 455-465.

Gryllos, I., Cywes, C., Shearer, M. H., Cary, M., Kennedy, R. C. \& Wessels, M. R. (2001). Regulation of capsule gene expression by group A streptococcus during pharyngeal colonization and invasive infection. Mol Microbiol 42, 61-74.

Gryllos, I., Levin, J. C. \& Wessels, M. R. (2003). The CsrR/CsrS twocomponent system of group A Streptococcus responds to environmental $\mathrm{Mg}^{2+}$. Proc Natl Acad Sci U S A 100, 4227-4232.

Guédon, E., Serror, P., Ehrlich, S. D., Renault, P. \& Delorme, C. (2001). Pleiotropic transcriptional repressor CodY senses the intracellular pool of branched-chain amino acids in Lactococcus lactis. Mol Microbiol 40, 1227-1239.

Guédon, E., Sperandio, B., Pons, N., Ehrlich, S. D. \& Renault, P. (2005). Overall control of nitrogen metabolism in Lactococcus lactis by CodY, and possible models for CodY regulation in Firmicutes. Microbiology 151, 3895-3909.

Gusa, A. A., Froehlich, B. J., Desai, D., Stringer, V. \& Scott, J. R. (2007). CovR activation of the dipeptide permease promoter (PdppA) in group A streptococcus. J Bacteriol 189, 1407-1416.

Hynes, W. (2004). Virulence factors of the group A streptococci and genes that regulate their expression. Front Biosci 9, 3399-3433.

Klenk, M., Koczan, D., Guthke, R., Nakata, M., Thiesen, H.-J., Podbielski, A. \& Kreikemeyer, B. (2005). Global epithelial cell transcriptional responses reveal Streptococcus pyogenes Fas regulator 
activity association with bacterial aggressiveness. Cell Microbiol 7, $1237-1250$

Kreikemeyer, B., Boyle, M. D. P., Leonard Buttaro, B. A., Heinemann, M. \& Podbielski, A. (2001). Group A streptococcal growth phaseassociated virulence factor regulation by a novel operon (Fas) with homologies to two-component-type regulators requires a small RNA molecule. Mol Microbiol 39, 392-406.

Levdikov, V. M., Blagova, E., Joseph, P., Sonenshein, A. L. \& Wilkinson, A. J. (2006). The structure of CodY, a GTP- and isoleucine-responsive regulator of stationary phase and virulence in Gram-positive bacteria. J Biol Chem 281, 11366-11373.

Malke, H., Steiner, K., McShan, W. M. \& Ferretti, J. J. (2006). Linking the nutritional status of Streptococcus pyogenes to alteration of transcriptional gene expression: the action of CodY and RelA. Int $J$ Med Microbiol 296, 259-275.

Serror, P. \& Sonenshein, A. L. (1996). Interaction of CodY, a novel Bacillus subtilis DNA-binding protein, with the $d p p$ promoter region. Mol Microbiol 20, 843-852.
Shelburne, S. A., Sumby, P., Sitkiewicz, I., Granville, C., DeLeo, F. R. \& Musser, J. R. (2005). Central role of a bacterial two-component gene regulatory system of previously unknown function in pathogen persistence in human saliva. Proc Natl Acad Sci U S A 102, 16037-16042.

Shivers, R. P. \& Sonenshein, A. L. (2004). Activation of the Bacillus subtilis global regulator CodY by direct interaction with branchedchain amino acids. Mol Microbiol 53, 599-611.

Sonenshein, A. L. (2005). CodY, a global regulator of stationary phase and virulence in Gram-positive bacteria. Curr Opin Microbiol 8, 203-207.

Steiner, K. \& Malke, H. (2001). relA-independent amino acid starvation response network of Streptococcus pyogenes. J Bacteriol 183, 7354-7364.

Steiner, K. \& Malke, H. (2002). Dual control of streptokinase and streptolysin $S$ production by the covRS and fasCAX two-component regulators in Streptococcus dysgalactiae subsp. equisimilis. Infect Immun 70, 3627-3636. 